\title{
Study Reports: Pharmacokinetics
}

National Cancer Institute

\section{Source}

National Cancer Institute. Study Reports: Pharmacokinetics. NCI Thesaurus. Code C79278.

An account of pharmacokinetic data pertaining to a particular event. 